\title{
Beyond binaries in medical knowledge: a call for epistemological reflexivity
}

Além dos binarismos na educação e conhecimento médico: um chamado à reflexividade epistemológica

Más allá de los binarios en la educación y conocimiento médico: una llamada a la reflexión epistemológica

Sara Donetto ${ }^{1}$

Alan $\mathrm{Cribb}^{2}$

Prof. Seppilli draws attention to the impossibility of separating the biological from the socio-historical dimensions of any serious study of human processes. We wish to strongly endorse his argument for more integrated approaches. In particular we wish to lament the persistence of sharp distinctions between 'hard' and 'soft' forms of knowledge in undergraduate medical education and argue that attempts to address this dichotomy need to move beyond 'zero-sum' disputes about the correct 'proportions' of hard and soft and look for ways to transcend this distinction.

During two academic years of fieldwork in a UK medical school ${ }^{3}$ we found unhelpful epistemological dichotomies and polarisations of learning. Dividing practices that separated 'science' from experiential, psycho-emotional and sociocultural knowledge appeared over and again in the data. It was common, for the medical students we spoke to, to distinguish a more 'scientific' and a more 'human' aspects of their medical learning. These were usually represented as two poles of a continuum. Of course, 'good' doctors were expected to be able to place themselves somewhere more or less half-way between these poles; nevertheless the underlying conceptualisation of medical knowledge assumed 'scientific facts' existed in a separate domain of learning from the 'fluffy' one in which communication and psychosocial factors belonged. 'Knowledge' for medical students had an almost concrete, solid, quantifiable character; 'to have a lot of your science' (Milele, Year-2) was the foundation of being a good doctor, whilst communication 'skills' were a useful complement to this knowledge.

Areas of knowledge were hierarchically positioned by students as a function of the extent to which they were seen as 'definitive' in shape. For example, many students were ambivalent about ethics. Whilst a few thought it provided useful reminders of the importance of respecting different beliefs and practices ${ }^{4}$, they were concerned that it lacked 'a bottom line': 'ok these are all the things we have to think about but in this country this is what we do. This is what's written in the law' (Sandro, Year-2 equivalent).

This discomfort with forms of learning unaccompanied by concrete protocols for practice pointed to a more general discomfort with uncertainty, fuzzy boundaries, and non-linear explanatory models. Ethics teaching encourages forms of explorative, open-ended thinking which are seen as uncomfortable. This
${ }^{1,2}$ Centre for Public Policy Research, Dept. of
Education and

Professional Studies, King's College London. Franklin Wilkins Building, Waterloo Road, London SE19NH. sara.donetto@kcl.ac.uk

3 Between 2005 and 2007, SD carried out extensive observations of various teaching settings in the medical school (workshops, lectures, tutorials, small group sessions) and semistructured interviews with 30 students and 11 educators (Donetto, $\mathrm{S}$. Critical awareness in undergraduate medical education: an ethnographic study of one UK medical school. 2009. Unpublished PhD thesis). For further details on the methodological approach of this study, see Donetto, S. (2010) Medical students' views of power in doctorpatient interactions: the value of teacher-learner relationships. Medical Education, 44:187-196. 
${ }^{4}$ See, for example, Barbara (Year-2): 'I mean, I can be aware of people's views and opinions [...] I know that helps me in being more open minded and not judging a patient and all that there, but you know, how does that work when it comes to making decisions for them, or they're making decisions for themselves and me not agreeing with it, or...? You know, how does that work?'

${ }^{5}$ See for example, Liang's (Year-4) comment: In terms of our learning, that is true, you do have to keep things separate. [...] And that's what I find quite fascinating about psychiatry. At the same time, the body is linked to the mind, it's also a completely different thing, and something you can study by itself

${ }^{6} \mathrm{GMC}$, General Medical Council (2003). Tomorrow's doctors. London: GMC; DoH, Department of Health (2004). Medical schools: delivering the doctors of the future. London: DoH, Crown Copyright. discomfort was even more marked in the ways in which students talked about mental health and psychiatry. Psychiatry, one student explained, was an unpopular discipline: 'because if you're a student you want things simple. You want ....signs, symptoms, organic signs and symptoms and a proper diagnosis... whereas psychiatry it's a lot more ... understanding and, you really have to acquire the skill of getting into people's minds and finding out what the matter is.' (Liang, Year-3) More generally, people suffering from mental illness were frequently perceived by students as 'unpredictable', and symptoms of mental illness were considered difficult to explain 'scientifically' and even more difficult to relate to. For students in later years it was not unusual to articulate a clear distinction between the physical and the mental ${ }^{5}$. Students' habit of somehow separating the psychological aspects of human suffering from the physical ones was also evident in the ways they sometimes conceptualised disease as a thing in itself, operating a distinction between 'the disease and the person' (e.g. James, Year-5; Sandro, Year-2 equivalent).

At least on the basis of these data, integrated approaches to the human experience do not seem to have found fertile ground in health professional education. The lack of more integrated conceptualisations of the learning and practice of medical knowledge(s) has important consequences in terms of the reproduction and circulation of hegemonic medical discourses.

We want to emphasise here that the sort of distinctions we reported can be seen as an indication of a deeply rooted issue about the epistemological hierarchies that still underpin medical education cultures. These hierarchies tend to position biomedical science as a superior, more valid, form of knowledge to all others, undermining the educational discourses that promote person-centred health care practice, collaborative approaches to clinical interactions and more authentic dialogue in medical work ${ }^{6}$.

More integrated understandings cannot be achieved solely through curriculum changes that follow a zero-sum model, i.e. by which more curricular space is allocated to 'soft' subjects in order to complement the 'hard sciences' teaching. Rather, we argue, change needs to take place through active questioning of this very distinction. We would suggest that the kind of epistemological reflexivity that is evident in Seppilli's account needs to become a pervasive and mundane feature of medical schools, such that learners, teachers and researchers are made routinely conscious of the ways in which they construct and classify forms of knowledge. The called for shift would be broadly analogous to the one that has gradually happened in relation to classifications and stereotypes around gender and 'race'. Once again the task is to recognise but not reify differences; and to seek practices that embody genuinely equal respect by challenging classifications, separations and hierarchies that are constructed as 'natural'. 